\title{
A produção científica mundial sobre Competência em Informação: análise dos documentos indexados na Web of Science
}

\author{
Djuli Machado De Lucca \\ Doutora; Universidade Federal de Rondônia. Porto Velho, RO, Brasil; \\ djuli.mdl@gmail.com \\ Patricia da Silva Neubert \\ Doutora; Universidade Federal de Santa Catarina Florianópolis, SC, Brasil; \\ patyneubert@hotmail.com
}

\begin{abstract}
Resumo: Trata-se de pesquisa que compreende a mensuração da produção científica sobre competência em informação em nível mundial, oportuna para a obtenção de indicadores acerca da atividade científica da temática. Analisa a produção científica em competência em informação indexada na Web of Science no período de 1957 a 2017. Tem como objetivos a) identificar os padrões da produção científica sobre competência em informação, no que se refere à modalidade de publicação e modelos de acesso, ano, citações, idioma, área e veículos de publicação e, b) identificar a origem dos documentos publicados, baseado na autoria, no vínculo institucional e na nacionalidade dos autores. $\mathrm{O}$ universo da pesquisa é composto pelos documentos indexados na Web of Science sobre competência em informação, em todos os formatos e idiomas publicados até 2017, totalizando 6.434 documentos. Os resultados demonstram o crescimento exponencial do número de documentos da temática na última década, que concentra a grande maioria dos trabalhos publicados $(74,85 \%)$, com o predomínio de artigos $(62,69 \%)$ publicados em língua inglesa $(90,43 \%)$, nas áreas de Ciência da Informação (34,96\%) e Educação (34\%), por autores de 112 países. Por fim, sinaliza a expansão numérica e geográfica no volume de documentos sobre Competência em Informação na Web of Science, indicando o desenvolvimento das pesquisas sobre o tema no cenário internacional.
\end{abstract}

Palavras-chave: Competência em Informação. Produção Científica. Bibliometria. Web of Science.

\section{Introdução}

A competência em informação (CoInfo) é um fenômeno social que surgiu na década de 1970, no cenário da sobrecarga informacional experienciada no período. Trata-se do conjunto de habilidades, atitudes, valores e conhecimentos 
(DUDZIAK, 2001; 2003) relacionados ao uso consciente, criativo e benéfico de recursos informacionais (VITORINO; PIANTOLA, 2009) que o sujeito desenvolve para alcançar a autonomia, o empoderamento social, a liberdade e a cidadania (AMERICAN LIBRARY ASSOCIATION, 1989; INTERNATIONAL FEDERATION OF LIBRARIES ASSOCIATIONS AND INSTITUTIONS, 2005).

Esse fenômeno também é investigado no campo científico. Pesquisas sobre CoInfo buscam explorar conceitos e definições, e ainda, apresentar práticas que podem ser desenvolvidas em determinados grupos de indivíduos. Os modelos para o desenvolvimento da CoInfo, que são estratégias delineadas para grupos específicos de pessoas que possuem características cognitivas, funcionais e ocupacionais específicas, foram criados a partir de pesquisas dessa natureza, e são instrumentos primordiais para a promoção de práticas com populações específicas. Tais investigações científicas oferecem, dessa forma, subsídios para que o movimento obtenha êxito na proposta para o desenvolvimento de tais capacidades. A partir dessa interação dinâmica, é possível observar que o avanço da CoInfo enquanto disciplina científica possui relação direta com o sucesso do movimento em âmbito social.

Análises da atividade científica da temática permitem que se demonstre a relação entre o desenvolvimento social e o acadêmico do movimento. A mensuração dessa atividade, por meio de indicadores, é útil para estabelecer relações entre a evolução da ciência e progresso social (MACIAS-CHAPULA, 1998). Realizar essa tarefa para avaliar tais aspectos da CoInfo é oportuno, dessa forma, para identificar o estado atual de tal disciplina científica que, por sua vez, pode refletir o desenvolvimento social da CoInfo enquanto fenômeno. A bibliometria, concebida por Macias-Chapula (1998) como o estudo dos aspectos quantitativos da produção, disseminação e uso da informação registrada por meio do desenvolvimento de padrões e modelos matemáticos, mostra-se útil para a consecução dessa tarefa.

Estudos acerca da produção científica sobre a temática vêm sendo realizados com o intuito de analisar o desenvolvimento da CoInfo desde a delimitação do campo, ocorrida em meados da década de 1970. São estudos que 
se dedicam a universos distintos, como a produção indexada em bases internacionais, como Scopus (BHARDWAJ, 2017; MAJID et al., 2015), e WoS (KOLLE 2017), ou em ambas as bases (DUDZIAK, 2010), cobrindo períodos temporais, específicos, geralmente centrados em análises a partir dos anos 2000 - de 2000 a 2009 (DUDZIAK, 2010), de 2001 a 2012 (BHARDWAJ, 2017), de 2003 a 2012 (MAJID et al., 2015), e de 2005 a 2014 (KOLLE, 2017), por exemplo, sem contemplar a evolução temporal da produção científica desde a delimitação do campo.

De acordo com Bhardwaj (2017), as análises quantitativas da literatura sobre CoInfo são escassas, o que dificulta a identificação dos padrões de evolução das pesquisas nesta temática. Por compreendermos que as análises dos padrões da produção científica indexada em bases de dados fornecem uma perspectiva histórica sobre a evolução e o panorama de um campo de pesquisa, este estudo se propõe a analisar a produção científica mundial em CoInfo indexada na Web of Science (WoS). Os objetivos deste estudo são:

a) identificar os padrões da produção científica sobre CoInfo, no que se refere à tipologia de documentos e seu modelo de acesso, ano, citações, idioma, área e veículos de publicação;

b) identificar a origem dos documentos publicados, com base no vínculo institucional dos autores.

\section{A trajetória da CoInfo enquanto disciplina científica}

A Competência em Informação enquanto disciplina científica acompanha sua evolução histórica enquanto movimento social: surge em contexto voltado às habilidades requeridas para o mundo do trabalho nos Estados Unidos (ZURKOWSKI, 1974) e, durante seu desenvolvimento, passa também a contemplar discussões para elementos como autoridade, desigualdade nos fluxos informacionais e intersubjetividade no processo de desenvolvimento dessa capacidade (ASSOCIATION OF COLLEGE AND RESEARCH LIBRARIES, 2016), direcionando suas pesquisas também para países em desenvolvimento. A trajetória da disciplina nas Ciências Sociais Aplicadas - particularmente a Ciência da Informação (CI) - também possui relação com tal evolução: inicia 
recebendo contribuições de áreas tradicionalmente consolidadas das Ciências Sociais Aplicadas e, no decorrer do seu desenvolvimento, passa a receber contribuições de áreas das Ciências Humanas, Sociais e Exatas, consolidando seu caráter multidisciplinar.

A década de 1970 constituiu-se como um cenário de mudança nas estruturas sociais, econômicas e políticas na sociedade. Parte dessas mudanças tem a informação enquanto elemento central. Em termos amplos, observa-se, naquele período, o reconhecimento dos fenômenos, processos, e atividades de informação enquanto um plano constitutivo das atividades e manifestações econômicas, sociais e culturais (GONZÁLEZ DE GÓMEZ, 2002). Em associação, verifica-se, ainda, a emergência das denominadas Tecnologias da Informação e Comunicação (TIC), popularmente restritas, em tal período, ao rádio e à televisão.

Esse cenário ofereceu elementos para a eclosão de um fenômeno descrito por Saracevic (1996) como um problema crítico: a sobrecarga informacional. Zurkowski (1974), por sua vez, narra que tal conjuntura fez emergir uma multiplicidade de rotas de acesso e fontes de informação para responder às necessidades informacionais dos indivíduos. Ainda, o autor mencionou, naquele período, que tais rotas eram "pobremente compreendidas e amplamente subutilizadas" (ZURKOWSKI, 1974, p. 4, tradução nossa). A CoInfo, desse modo, surgiu do problema social da sobrecarga informacional aliado à utilização precária e irreflexiva dos recursos e fontes de informação disponíveis.

O termo Competência em Informação - em inglês, information literacy foi designado pela primeira vez no ano de 1974 pelo bibliotecário americano Paul Zurkowski. No documento intitulado 'The information service environment: relationships and priorities', Zurkowski (1974) designa a CoInfo como o conjunto de técnicas e habilidades para utilizar a vasta gama de recursos de informação na solução de problemas informacionais (ZURKOWSKI, 1974). A CoInfo, nessa perspectiva, envolve o reconhecimento do valor da informação e a habilidade de ajustar a informação para atender a distintas necessidades de informação no contexto de explosão informacional (ZURKOWSKI, 1974). 
Com um apelo social característico, o documento escrito por Zurkowski (1974) foi útil para o reconhecimento da relevância da CoInfo em âmbito científico: ainda na década de 1970, é possível compreender a emergência de uma vertente de pesquisa acerca da temática. Pinto, Cordón e Díaz (2010) apontam os trabalhos de Burchinal (1976) e Hamelink (1976) e que, segundo a autora, buscaram solidificar os benefícios da CoInfo na sociedade da informação, na medida em que expuseram seus elementos para a emancipação política e para a resolução de situações rotineiras do dia a dia.

Na década de 1980, também é possível observar um corpo de pesquisas sobre CoInfo: Dudziak (2003) indica que, a partir da emergência das TIC nas rotinas de bibliotecas, autores como Breivik (1985) e Kuhlthau (1987) expuseram acerca da necessidade de integrar a CoInfo nos currículos de cursos de graduação e solidificar um modelo de aprendizagem centrado em recursos de informação. Ainda na mesma década, é publicada a Declaração da American Library Association (ALA) intitulada 'Presidential Committee on Information Literacy: Final Report', que expressa a importância designada a tal movimento pela organização. A declaração é um dos documentos mais populares na literatura científica do campo (DUDZIAK, 2003) e expõe a CoInfo como um elemento para a promoção da autonomia e empoderamento pessoal dos sujeitos, na medida em que permite que cada um possa “[...] construir seus próprios argumentos e experimentar a emoção na busca pelo conhecimento." (AMERICAN LIBRARY ASSOCIATION, 1989, p. 1, tradução nossa).

$\mathrm{O}$ período que compreende a última década do século $\mathrm{XX}$ foi, segundo Pinto, Escalona-Fernández e Pulgarín (2013), marcado pela solidificação do conceito de CoInfo. Dudziak (2003) menciona que, em tal período, a CoInfo foi popularizada entre os bibliotecários, e o papel desses profissionais enquanto educadores ganhou notoriedade. Em tal período, ainda, foi criado o Institute for information literacy da ALA, que era destinado prioritariamente a preparar os bibliotecários para o novo papel, além de dar suporte à implementação de programas educacionais no ensino superior (DUDZIAK, 2003). Esse cenário denota a popularização da CoInfo em âmbito social. No âmbito científico, não foi diferente: nessa fase, segundo a pesquisa desenvolvida por Pinto, Escalona- 
Fernández e Pulgarín (2013), observou-se um crescimento exponencial da produção científica de CoInfo.

Na década de 2000, há continuidade do crescimento exponencial das pesquisas científicas na temática. A implementação da CoInfo nas instituições tornou-se prioridade em organizações como a International Federation of Library Associations and Institutions (IFLA) e a United Nations Educational, Scientific and Cultural Organization (UNESCO), que passaram a idealizar e/ou apoiar encontros e conferências específicas sobre o tema em todos os continentes (PINTO; ESCALONA-FERNÁNDEZ; PULGARÍN, 2013). Ainda, o movimento começou a ser socializado com outros profissionais além dos bibliotecários, e a promoção da CoInfo passou a receber a colaboração de educadores, acadêmicos e profissionais de outros campos do saber.

No campo científico, tal movimento também se intensificou: a diversidade de documentos expandiu-se, agregando teses e dissertações que apresentam modelos, ferramentas, aplicações e programas para o desenvolvimento da CoInfo, e, ainda, ocorreu a emergência de estudos com abordagem multi-focalizada, que receberam contribuições de administradores, engenheiros, psicólogos, biólogos, dentre outras áreas (PINTO; ESCALONAFERNÁNDEZ; PULGARÍN, 2013). Os autores também relatam um movimento relevante: a partir dessa década, as pesquisas, que antes estavam concentradas em países como Estados Unidos da América (EUA), Canadá, Reino Unido, Austrália e Nova Zelândia, passaram a ser desenvolvidas também em países como China, Alemanha, México, Escandinávia, Singapura, África do Sul, América do Sul, Espanha, dentre outros, denotando a expansão da disciplina também em termos geográficos.

$\mathrm{Na}$ segunda década do século XX, tal tendência permanece: Kolle (2017), que realizou um estudo bibliométrico sobre a temática no período de 2005 a 2014, observou um "Aumento consistente da literatura em competência em informação [...]" (KOLLE, 2017, p. 296, tradução nossa). Majid et al. (2015) analisaram as tendências de pesquisa e apontaram que as investigações, a partir dessa década, se concentraram mais em resultados de pesquisas aplicadas, em detrimento aos estudos teoréticos. Tal afirmação indica, dessa forma, o 
amadurecimento da CoInfo enquanto disciplina científica, aspecto que indica a consolidação de linhas de pesquisa, de pesquisadores e veículos de comunicação especializados na temática. Dessa forma, estudos acerca da produção científica são úteis à compreensão do comportamento e desenvolvimento do campo.

\section{CoInfo: características da produção científica}

A CoInfo enquanto campo de estudo surgiu em 1974, com a publicação do documento de Paul Zurkowski (1974). Trata-se de uma vertente de pesquisa bastante explorada na literatura: a pesquisa de Rader (2002), por exemplo, identificou, nos primeiros trinta anos do movimento, um montante de mais de 5.000 publicações acerca da CoInfo e educação de usuários. O autor ainda salienta que isso indica um "crescimento fenomenal" de uma disciplina científica (RADER, 2002). Na quarta década de tal disciplina, também é possível observar o crescimento exponencial: Kolle (2017) menciona que, entre os anos de 2005 a 2014, o número de publicações científicas identificadas expandiu: na pesquisa desenvolvida pelo autor, que contemplou resultados da WoS, o número de documentos saltou do montante de 66 artigos, no ano de 2005, para 210 artigos, em 2014.

Por tratar-se de um tema multidisciplinar, a identificação das áreas em que se realizam pesquisas sobre CoInfo é uma questão oportuna para explorar. A pesquisa de Majid et al. (2015), que investigou tal disciplina entre os anos de 2003 a 2012, identificou que, na base de dados Scopus, 57,4\% de todas as publicações sobre a temática foram desenvolvidas na grande área das Ciências Sociais. De modo específico, Dudziak (2010) investigou as publicações da referida disciplina entre os anos 2000 a 2009 nas bases WoS e Scopus, e identificou que a área específica do saber em que mais se concentram tais estudos é a Ciência da Informação. Na ocasião, Dudziak (2010) observou que mais de metade $(52 \%)$ de todos os documentos publicados sobre a temática naquele período estavam concentrados na área. Kolle (2017) também observou resultados semelhantes: sua pesquisa, desenvolvida a partir dos artigos publicados sobre o tema na WoS entre os anos de 2005 a 2014, verificou que 
49,9\% dos documentos estavam concentrados no campo da Ciência da Informação.

Consideramos axiomática a relação entre Ciência da Informação e CoInfo: desde a gênese, as duas esferas possuem características em comum. Ambos os movimentos têm sua origem relacionada à sobrecarga informacional, um atributo da revolução tecnológica, e buscam encontrar soluções para atenuar seus impactos na sociedade. O propósito também é comum para ambas: a Ciência da Informação trata "[...] dos problemas de efetiva comunicação de conhecimentos e de registros do conhecimento entre seres humanos, no contexto de usos e necessidades sociais, institucionais e/ou individuais de informação." (SARACEVIC, 1996, p. 2). Os estudos sobre CoInfo, por sua vez, estão voltados para a investigação das

[...] habilidades, atitudes e compreensão necessárias para identificar, avaliar e usar a informação, de acordo com as necessidades de informação de cada indivíduo, em estruturas formais ou não de informação. (LEITE et al., 2016, p. 153).

A congruência entre as duas esferas é evidenciada na ocasião em que se busca mutuamente compreender, descrever ou analisar situações informacionais individuais ou coletivas.

Além disso, é possível verificar a contribuição de outras áreas do conhecimento para as pesquisas sobre CoInfo. A pesquisa desenvolvida por Dudziak (2010) identificou que, dos documentos indexados entre os anos 2000 a 2010 na WoS, 22\% deles estão concentrados nas áreas de Ciência da Computação e Sistemas de Informação. A autora também observou a contribuição da área da Educação, que apresentou 9\% dos registros recuperados. Já a pesquisa de Kolle (2017), que compreendeu documentos indexados na mesma base no período de 2005 a 2014, identificou a presença relevante da área da Educação: 21.8\% de todos os registros pertenciam a tal área do conhecimento. A pesquisa também identificou a contribuição da área de Ciência da Computação, que compreendeu $11.8 \%$ dos registros, e da Comunicação, que concentrou $10.8 \%$ dos resultados. Dessa forma, é possível compreender que, embora a Ciência da Informação seja hegemônica na apresentação dos 
resultados, observa-se a presença de outros campos do saber nas pesquisas, indicando, mesmo que de forma sutil, elementos de multidisciplinaridade na constituição e na produção do campo.

Ainda, a expansão da CoInfo enquanto disciplina científica também pode ser observada por meio da distribuição geográfica das publicações. Bhardwaj (2017), que realizou análise bibliométrica das publicações sobre CoInfo indexadas na base de dados Scopus entre os anos de 2001 a 2012, observou que naquele período as pesquisas estavam distribuídas em 79 países. Destes, 13 países publicaram mais de $1 \%$ do total de publicações, enquanto os outros 66 concentraram menos de $1 \%$ do total cada um. Bhardwaj (2017) identificou a presença massiva dos EUA, que é o 'berço' do movimento da CoInfo, tanto em âmbito científico quanto social. O autor também verificou a contribuição expressiva de outros países: O Reino Unido, por exemplo, concentra 7,7\% do total de artigos recuperados, o Canadá, 5,0\%, e a Austrália, 4.4\%. Em seguida, registram-se contribuições da Espanha, de Taiwan, e da China, concentrando $3,1 \%, 1,9 \%$ e $1,8 \%$ dos documentos, respectivamente. Contribuem, também com mais de $1 \%$ dos resultados, a África do Sul (1,5\%), a Nova Zelândia (1,3\%), Hong Kong (1,1\%), Malásia (1,1\%) e Nigéria (1,0\%).

Majid et al. (2015) também analisaram a distribuição geográfica das publicações. A pesquisa desenvolvida pelos autores, que observou o movimento científico da CoInfo entre os anos de 2003 a 2012, identificou a contribuição expressiva dos EUA, que concentrou $47,4 \%$ de todas as publicações sobre a temática. Majid et al. (2015), ainda, verificaram presença relevante de países como o Reino Unido (7,4\% do total de publicações) e o Canadá (5,7\% dos documentos recuperados). Contribuições de outros países também foram observadas: Austrália (4,6\%), China (3,6\%), Espanha $(2,9 \%)$, Taiwan $(2,6)$, África do Sul (1,4\%), Nova Zelândia (1,3\%), Japão (1,2\%), Iran (1,1\%), Hong Kong $(1,1 \%)$ e Suécia $(1,1 \%)$ também apresentaram mais de $1 \%$ do total de resultados cada um.

As pesquisas publicadas por Majid et al. (2015) e Bhardwaj (2017), que apresentam resultados de pesquisa relativamente recentes, trazem a conotação de que a CoInfo continua, ainda no século XXI, sendo explorada com 
veemência nos EUA. É possível observar também um movimento - ainda que sutil, de popularização da disciplina em outros países e regiões do globo.

\section{Procedimentos metodológicos}

Para análise da produção científica sobre CoInfo indexada na WoS, este estudo adota uma abordagem quantitativa, amparada em análises bibliométricas. O universo da pesquisa é composto pelos documentos sobre a temática indexados na Coleção Principal da base de dados WoS.

A estratégia de busca utilizada para a recuperação dos documentos consistiu na consulta pelo equivalente do termo em língua inglesa, combinados aos termos utilizados nos estudos de Bhardwaj (2017) e Kolle (2017), na opção de pesquisa avançada no campo Tópico (TS= "information literacy" OR "information literacies" OR "media literacy" OR "digital literacy" OR "meta literacy" OR "meta literacies" OR "information skills instructions" OR "information learning" OR "information resources literacy" OR "critical literacy" OR “critical literacies"), que abrange o título, resumo, as palavraschave de autor e Keywords Plus® (CLARIVATE ANALYTICS, 2018), em todos os formatos de documentos e idiomas, estipulando o tempo de publicação de 1945, início da cobertura da base de dados, até 2017. A consulta foi realizada em julho de 2018 e foram identificados 6.434 documentos.

O download dos resultados da busca foi realizado em lotes de 500 registros em formatos .txt, limitados pela base de dados e, tais resultados foram unificados em arquivo único e processados em base de dados relacional elaborada no software Excel®. A contabilização dos documentos emprega o método de contagem completo, sendo realizado o fracionamento dos dados, respeitando as sobreposições identificadas, para as análises por país e área do conhecimento, assim, contabilizando as nacionalidades e as áreas de pesquisa tantas quantas fossem suas menções em cada documento. Para identificar os trabalhos mais citados, foi utilizada a contagem do número de citações da Principal Coleção do WoS (campo TC). A identificação das áreas do conhecimento nas quais os documentos foram classificados segue a categorização adotada em WoS (campo SC - áreas de pesquisa). 
Entre as limitações do estudo está a cobertura da base, limitada aos documentos indexados na WoS, uma vez que a base não cobre todos os títulos e fontes de informação publicadoras de documentos sobre CoInfo.

\section{Análise e discussão dos resultados}

Foram publicados e indexados na WoS 6.434 documentos sobre CoInfo no período coberto pela pesquisa. Os artigos são o formato de publicação mais utilizado dentre os itens, 4.033 do total $(62,69 \%)$ e são seguidos pelos trabalhos publicados em eventos (Proceedings Paper), 1.783 (27,72\%). Juntos, estes formatos totalizam 90,39\% dos documentos da temática até 2017. Aparecem na terceira posição Book Review (5,53\%), seguido de Review (2,35\%), e Editorial Material (2,27\%), de acordo com a Figura 1.

Figura 1 - Tipologia dos documentos sobre CoInfo indexados em WoS por modelo de acesso

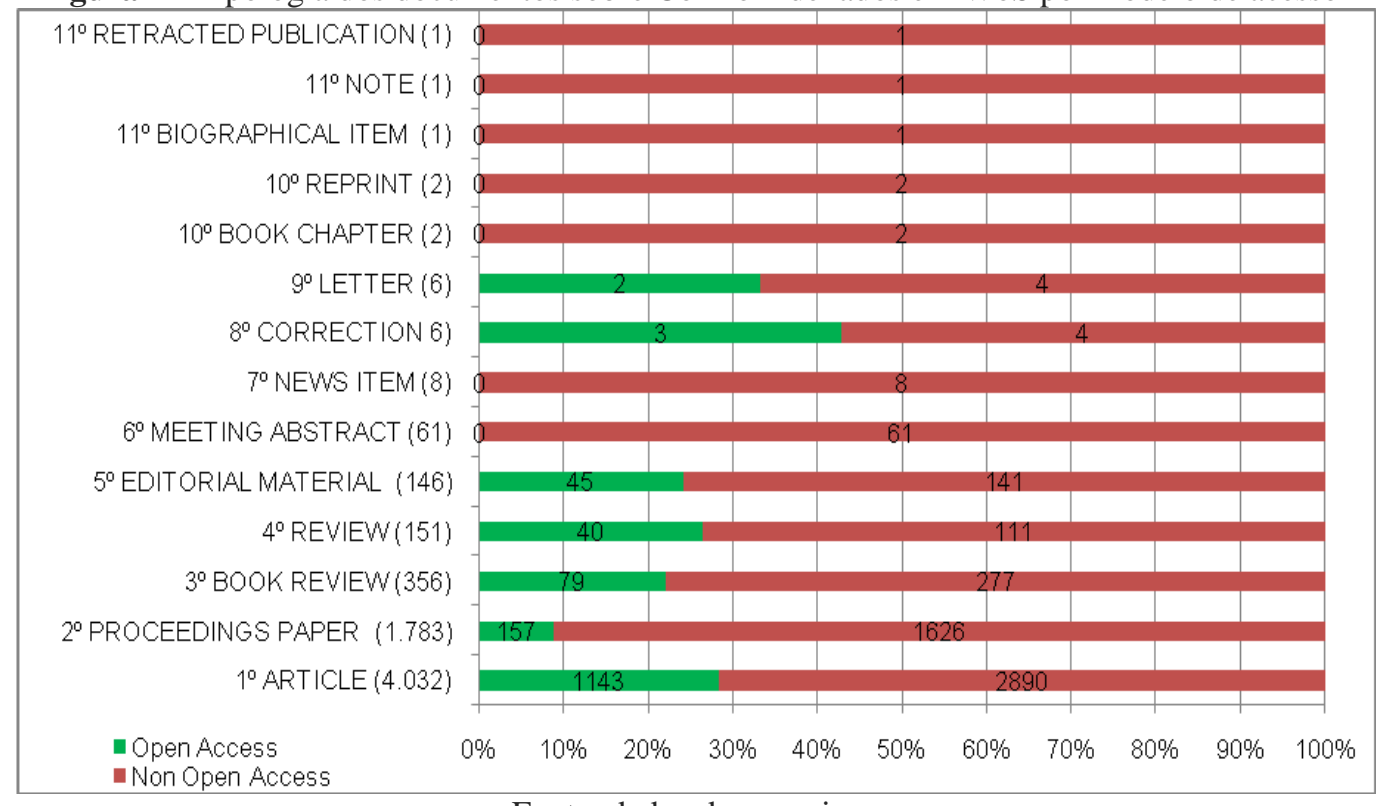

Fonte: dados da pesquisa.

Resultados similares quanto à prevalência dos artigos de periódicos também foram observados por Bhardwaj (2017), Kolle (2017), Majid et al. (2015), Kumari, Madhusudhan e Ali (2015), Kumar (2014), Aharony (2010) e Dudziak (2010), embora nos resultados dos estudos de Bhardwaj (2017) e Kumari, Madhusudhan e Ali (2015) seja possível observar a inversão dos 
documentos de eventos da segunda para a terceira colocação, enquanto na segunda posição aparecem Review Papers e Book Review, respectivamente.

Apesar de haver evidente destaque dos documentos publicados em periódicos e anais de eventos, pode-se verificar que há outros formatos indexados na base: Meeting Abstract (0,95\%), News item (0,12\%) Correction (0,11\%), Letter (0,09\%), Book Chapter e Reprint (0,03\% cada) e Biographical item, Note e Retracted Publication (0,02\% cada). E são documentos cuja forma de acesso majoritariamente é feita por assinatura (57,14\% Correction, 66,67\% de Letter, 71,68\% de Article, 73,51\% de Review, 77,81\% de Book Review, 91,19\% dos Proceedings Paper, 96,57\% de Editorial material e 100\% dos demais tipos documentais). Dos formatos que concentram o maior volume de documentos, apenas 28,34\% (1.143) dos artigos e, 8,80\% (157) dos Proceedings Paper estão disponíveis em acesso aberto (Figura 1).

Embora existam registros de documentos indexados desde $1957^{1}$, é a partir dos anos 1980 que é possível observar uma constância nas publicações sobre CoInfo. Nesta década as publicações são reduzidas (15) e intervaladas nos primeiros anos, mas a partir de 1985, WoS registra a existência de publicações anuais (Figura 2). É nesta década que o movimento de CoInfo começa a ser impulsionado pelo crescente uso das TICs e pela declaração da ALA (DUDZIAK, 2003). 
Figura 2 - Documentos sobre CoInfo indexados na WoS por ano

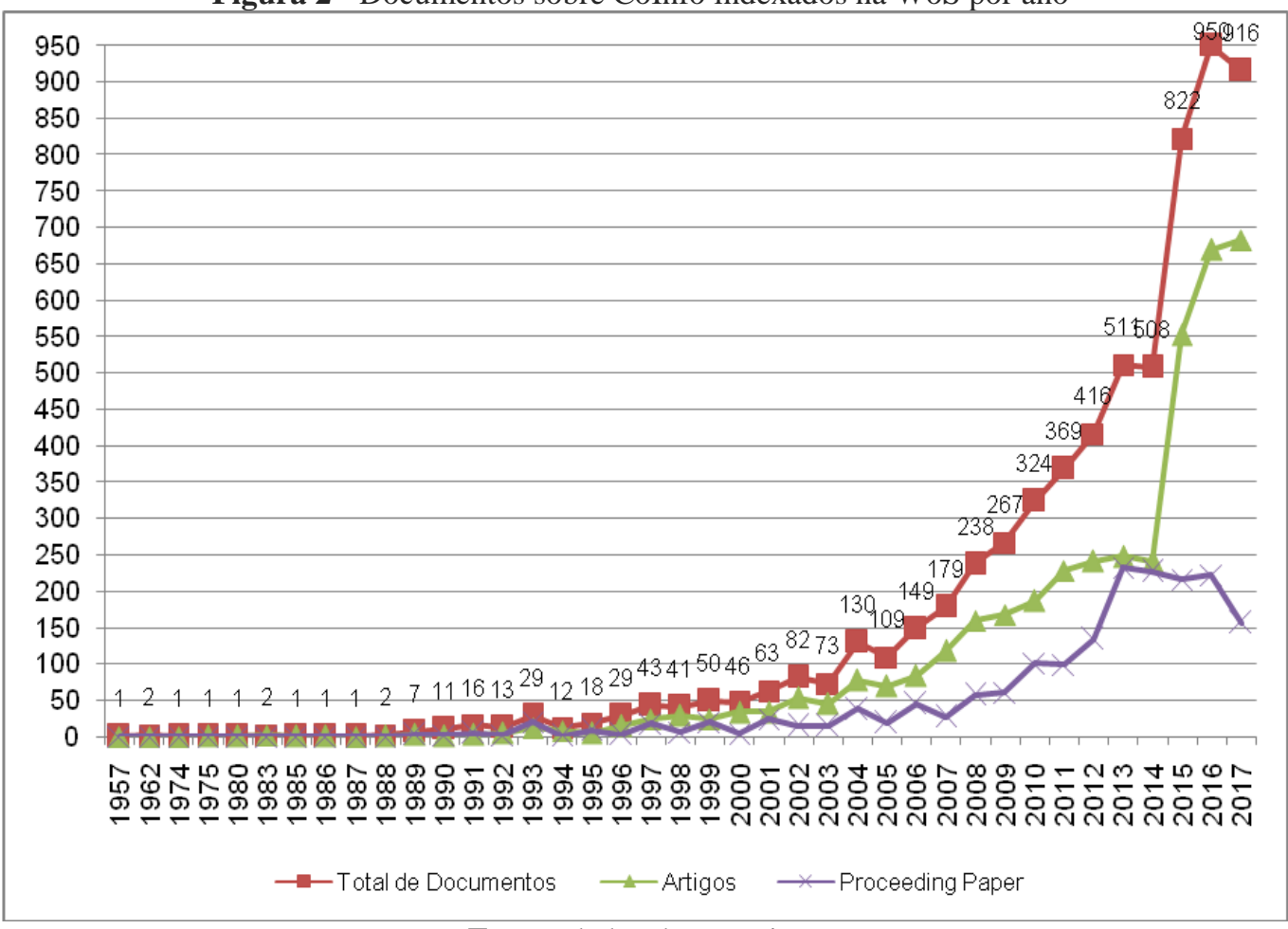

Fonte: dados da pesquisa.

A partir da década de 1990, é possível observar uma maior constância e o adensamento no volume de documentos publicados anualmente sobre a temática, de 11 documentos em 1990 para 50 em 1999. Neste período, há a consolidação do conceito de CoInfo (PINTO; ESCALONA-FERNÁNDEZ; PULGARÍN, 2013), o que explica o crescimento do número de publicações.

Após os anos 2000, é possível observar uma mudança no volume de publicações ao observar a curva de crescimento de documentos indexados sobre CoInfo (de 46 em 2000 a 916 em 2017). Em meados dessa década o número de publicações anuais ultrapassa uma centena (130 em 2004) e segue um crescimento constante, resultando em $20,77 \%$ do total de documentos publicados no período. Um crescimento similar é observado no estudo de González-Valiente (2014) sobre a produção científica cubana em CoInfo, e nos estudos de Dudziak (2010), Pinto, Escalona-Fernández e Pulgarín (2013) e Majid et al. (2015), cujos resultados reforçam o crescimento no volume de publicações na temática em meados dos anos 2000.

Porém, é a partir da década seguinte que, de acordo com o contabilizado até 2017 , o volume de produções reúne $74,85 \%$ das publicações na temática. O 
volume de documentos cresce significativamente, passando de 324 documentos em 2010 para 916 em 2017. Estes dados coincidem com a observação de que a pesquisa em CoInfo tem apresentado crescimento exponencial, desde a concepção do conceito, de acordo com Rader (2002), Kumari, Madhusudhan e Ali (2015) e Bhardwaj (2017).

Uma curva similar é observada no crescimento do número de citações anuais a estes documentos, inferior a 11 até 1994 e superior a 100 a partir de 2001 (147), chegando a 6.363 citações no ano de 2017 (Figura 3), totalizando (até julho de 2018) 38.804 citações entre os 6.434 artigos sobre CoInfo indexados em WoS, uma média de 6,03 citações por item, média superior a observada por Bhardwaj (2017), de cinco citações por documento.

Entretanto, é preciso considerar que um número expressivo de documentos, $3.050(47,40 \%)$, não possui sequer uma citação. Deste modo, o total de documentos citados pelo menos uma vez é de 3.384, o que aumenta a média de citações por documento citado para 11,47. Os trabalhos considerados mais citados (37), com o número de citações superiores a 100 (média de 149,78), foram publicados entre 1994 e 2014 e concentram 14,31\% (5.542) do total de citações.

Figura 3 - Número de citações anuais dos documentos (1958-2018)

Número de citações por ano

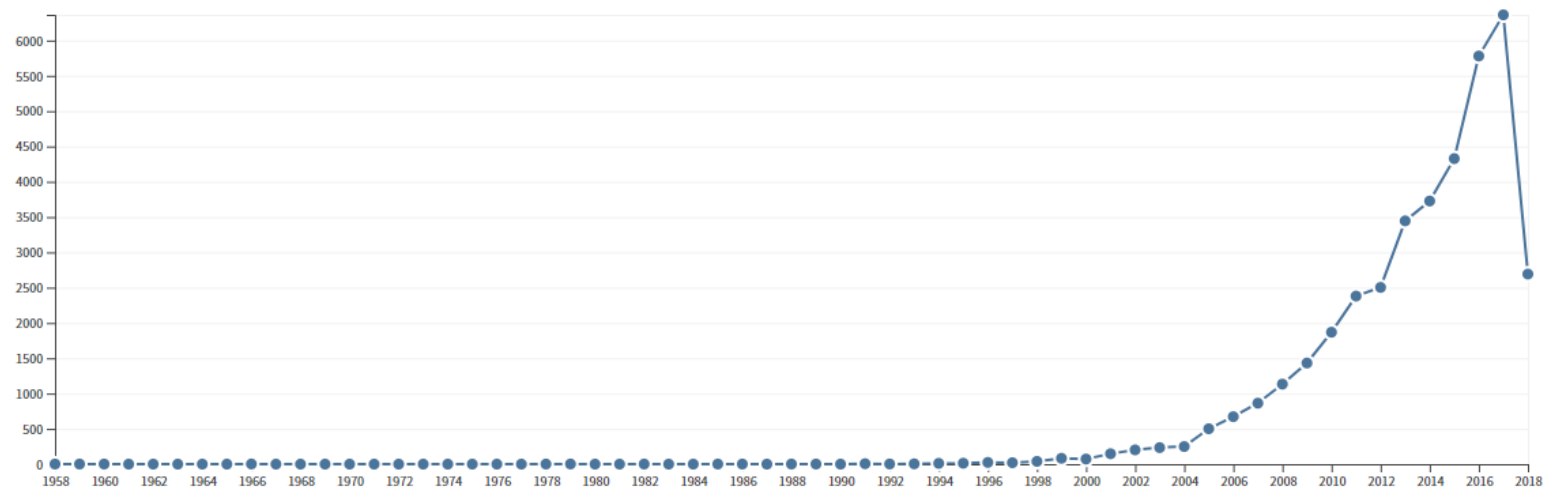

Fonte: Clarivate Analytics (2018). 
São registrados na WoS documentos em 24 idiomas diferentes, dos quais a maior parte (5.818), 90,43\%, é publicada em inglês, um reflexo da hegemonia exercida pela base na indexação de publicações neste idioma (KOLLE, 2017). Idiomas como o espanhol e o português representam, respectivamente, $4,8 \%$ e $1,46 \%$, das publicações. Uma variedade de outros idiomas aparecem com participação inferior a $1 \%$ cada: Russian $(0,96 \%)$, Chinese $(0,67 \%)$, German (0,42\%), Turkish (0,2\%), French (0,17\%), Japanese (0,16\%), Hungarian (0,12\%), Croatian (0,09\%), Dutch (0,09\%), Italian (0,08\%), Czech (0,08\%), Afrikaans (0,05\%), Slovenian (0,05\%), Latvian (0,03\%), Lithuanian (0,03\%) Polish (0,03\%), Slovak (0,03\%), Ukrainian (0,03\%), Bulgarian (0,02\%), Estonian (0,02\%) e Malay (0,02\%), que juntos somam 3,33\% do total.

Bhardwaj (2017) observa variedade linguística semelhante ao identificar a publicação da produção em CoInfo indexada em Scopus em 16 idiomas. Estudos anteriores também constatam o predomínio de documentos em inglês entre a produção em CoInfo indexada em bases multidisciplinares internacionais, sempre superiores a 90\% dos documentos indexados - 91,1\% (KOLLE, 2017), 92,70\% (KUMAR, 2014), 94,4\% (BHARDWAJ, 2017), 96,29\% (AHARONY, 2010).

Dentre a nacionalidade de origem das publicações, foram identificados 112 países (Tabela 1), o que comprova a expansão da disciplina em termos geográficos, como observado anteriormente por Pinto, Escalona-Fernández e Pulgarín (2013) e Majid et al. (2015). No entanto, 92 destes países contribuem com menos de $1 \%$ dos documentos indexados na base, dos quais 19 publicaram apenas 1 documento, 7 países publicaram 2, 26 países de 3 a 10 documentos e os demais (40 países) publicaram entre 12 e 63 documentos.

Tabela 1 - Ranking da nacionalidade dos documentos sobre CoInfo na WoS (1958-2017)

\begin{tabular}{c|l|c|c}
\hline & \multicolumn{1}{|c|}{ Países } & \multicolumn{2}{|c}{ Documentos } \\
\hline $1^{\circ}$ & EUA & 2.273 & $\%$ \\
$2^{\circ}$ & Espanha & 419 & $35,33 \%$ \\
$3^{\circ}$ & Inglaterra & 394 & $6,51 \%$ \\
$4^{\circ}$ & Austrália & 378 & $6,12 \%$ \\
$5^{\circ}$ & República Popular da China & 331 & $5,88 \%$ \\
$6^{\circ}$ & Canadá & 283 & $5,15 \%$ \\
& & & $4,40 \%$
\end{tabular}




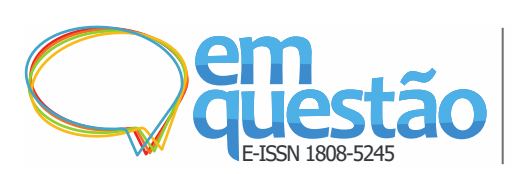

A produção científica mundial sobre Competência em informação: análise dos documentos indexados na Web of Science

Djuli Machado De Lucca e Patricia da Sillva Neubert

\begin{tabular}{c|l|c|c}
$7^{\circ}$ & África do Sul & 144 & $2,24 \%$ \\
$8^{\circ}$ & Brasil & 135 & $2,10 \%$ \\
$9^{\circ}$ & Alemanha & 127 & $1,97 \%$ \\
$10^{\circ}$ & Taiwan & 101 & $1,57 \%$ \\
$11^{\circ}$ & Suécia & 89 & $1,38 \%$ \\
$12^{\circ}$ & Turquia & 75 & $1,32 \%$ \\
$13^{\circ}$ & Cingapura & 74 & $1,17 \%$ \\
& Nova Zelândia & 74 & $1,15 \%$ \\
$14^{\circ}$ & Rússia & 73 & $1,15 \%$ \\
$15^{\circ}$ & Portugal & 71 & $1,13 \%$ \\
$16^{\circ}$ & Japão & 69 & $1,10 \%$ \\
$17^{\circ}$ & Itália & 66 & $1,07 \%$ \\
$18^{\circ}$ & Escócia & 65 & $1,03 \%$ \\
$19^{\circ}$ & Países Baixos & 5.326 & $82,01 \%$ \\
\hline \multicolumn{2}{|c|}{ Total } &
\end{tabular}

Fonte: Dados da pesquisa.

Nota: Percentual calculado em relação ao total de documentos sobre CoInfo (6.433). Considerados para ranqueamento apenas países com percentual superior a $1 \%$ do total de documentos.

A fim de analisar a nacionalidade da maioria dos documentos sobre CoInfo, foram considerados como os mais produtivos os países com participação superiores a $1 \%$ do total de documentos (Tabela 1). Notadamente, o país que possui autoria de mais de um terço dos documentos indexados em WoS sobre CoInfo é os EUA (35,33\%) o que também explica o predomínio do inglês entre os documentos indexados na base.

Bhardwaj (2017), Kolle (2017), Kumari, Ali e Madhusudhan (2015), Aharony (2010) e Dudziak (2010) também observaram o predomínio das contribuições norte-americanas entre as publicações indexadas na temática. $\mathrm{Na}$ segunda posição, em ambos os estudos, está a Inglaterra: aqui $3^{\text {a }}$ colocada (Tabela 1), seguida por Canadá e Austrália alternados entre a terceira e quinta posições destes rankings. A Espanha, segunda colocada neste estudo, aparece entre a quarta e quinta posição, exceto em Aharony (2010) e Dudziak (2010) no qual ocupa o sétimo lugar. Na Tabela 1, pode-se observar a ascensão de posição da Espanha. em comparação com os estudos anteriores e, diferente dos resultados já obtidos, Canadá registra queda de posição, ocupando a $6^{\mathrm{a}}$ posição neste estudo. Além disso, a República Popular da China, que não consta no ranking de Kumari, Ali e Madhusudhan (2015), ocupa a $7^{\mathrm{a}}$ posição no ranking 
de Bhardwaj (2017) e a $10^{\mathrm{a}}$ no estudo de Aharony (2010), sobe para a $5^{\mathrm{a}}$ colocação nesta pesquisa, mesma posição do ranking de Dudziak (2010). Deste modo, é possível observar o que Pinto, Escalona-Fernández e Pulgarín (2013) identificam como uma espécie de globalização dos estudos em CoInfo, com a variedade de nações, inclusive as consideradas periféricas ou em desenvolvimento, que passam a integrar o ranking de publicações na temática.

Kumari, Ali e Madhusudhan (2015) observam que a maioria dos artigos sobre CoInfo indexados na WoS se originam no continente americano $(52,43 \%)$. Neste estudo, os países do continente americano produzem 44,47\% dos documentos (EUA 2.273, Canadá 283, Brasil 135, México 53, Colômbia 37, Chile 18, Cuba 17, Equador 15, Argentina 9, Costa Rica 5, Uruguai 5, Venezuela 5, Peru 4, e Guatemala 2).

Ao estudar as áreas nas quais tais documentos são publicados, foram identificadas 117 áreas em que estes estão indexados na WoS. Entre estas, há três áreas do conhecimento que se destacam entre os documentos publicados sobre CoInfo. A área de Information Science, Library Science é a categoria que reúne o maior número de documentos sobre a temática, 2.249 (34,96\%), seguida pela área de Education, Educational Research, 2.187 (34\%) e pelos documentos categorizados em Computer Science, 971 (15,09\%).

Tabela 2 - Ranking das áreas de pesquisa das publicações sobre CoInfo na WoS (1958-2017)

\begin{tabular}{lcc}
\hline & \multicolumn{2}{c}{ Documentos } \\
\cline { 2 - 3 } \multicolumn{1}{c}{ Áreas de pesquisa } & n & $\%$ \\
\hline Information Science Library Science & 2.249 & $34,96 \%$ \\
Education Educational Research & 2.187 & $34,00 \%$ \\
Computer Science & 971 & $15,09 \%$ \\
Communication & 475 & $7,38 \%$ \\
Psychology & 286 & $4,45 \%$ \\
Engineering & 276 & $4,29 \%$ \\
Social Sciences Other Topics & 262 & $4,07 \%$ \\
Linguistics & 220 & $3,42 \%$ \\
Business Economics & 159 & $2,47 \%$ \\
Nursing & 97 & $1,51 \%$ \\
Public Environmental Occupational Health & 97 & $1,51 \%$ \\
Health Care Sciences Services & 94 & $1,46 \%$ \\
Chemistry & 67 & $1,04 \%$ \\
\hline
\end{tabular}

Fonte: dados da pesquisa. 
A relação entre CoInfo e estas áreas também foi observada por Dudziak (2010), ao constatar a concentração das publicações nas áreas de Ciências Sociais (58\%) e Ciência da Computação (15\%) na Scopus e, CI (52\%), Ciência da Computação (22\%) e Educação (9\%) na WoS. As duas primeiras colocadas neste estudo foram identificadas por Aharony (2010) como as categorias preferenciais de publicação dos documentos sobre CoInfo, seguidas pela área Public, Environmental and Occupational Health, $11^{\mathrm{a}}$ colocada neste estudo (Tabela 2).

Também foi observada a distribuição dos documentos entre as fontes de informação indexadas pela WoS. Foram identificadas 2.135 fontes publicadoras dos 6.434 documentos sobre CoInfo, uma média de 3,31 documento por veículo de publicação. No entanto, a distribuição dos dados revela parâmetros de concentração da produção científica sobre a temática, ao permitir a identificação de que a maioria absoluta das fontes, 2.017 das $2.135(94,47 \%)$ publica 56,45\% dos documentos (3.632 de 6.434): 1.355 apenas 1 documento, 319 publicam 2 documentos (totalizando 638 documentos) e 344, entre 3 a 10 (1.639 documentos).

O ranking das fontes que concentram o maior número de documentos é composto por publicações editadas nos países apontados como os editores da maioria das fontes de informação indexados em WoS: EUA, Alemanha, Inglaterra, Holanda, mais Espanha (Tabela 3). A primeira posição é do Journal of Academic Librarianship que também já foi identificado em outros estudos como fonte publicadora da maioria dos documentos sobre CoInfo (AHARONY, 2010; DUDZIAK, 2010; KUMARI; MADHUSUDHAN; ALI, 2015; KOLLE, 2017). Portal-Libraries and the Academy e College \& Research Libraries, apareceram nas posições 2 e 3 (DUDZIAK, 2010) e 3 e 4 (KUMARI; MADHUSUDHAN; ALI, 2015; KOLLE, 2017). 
Tabela 3 - Ranking das fontes de informação com a maior concentração de documentos sobre

\begin{tabular}{|c|c|c|c|c|c|c|}
\hline \multicolumn{7}{|c|}{$\begin{array}{rr}\text { CoInfo } \\
\end{array}$} \\
\hline \multirow{2}{*}{\multicolumn{2}{|c|}{ Título }} & \multirow{3}{*}{\begin{tabular}{|l} 
Tipos de documentos \\
Articles (175) \\
Book Review (37) \\
Review (7) \\
Editorial material (3) \\
Proceedings Paper (2) \\
Correction (1)
\end{tabular}} & \multirow{3}{*}{$\begin{array}{l}\text { País } \\
\text { EUA }\end{array}$} & \multirow{3}{*}{$\begin{array}{l}\text { Editora } \\
\text { Elsevier }\end{array}$} & \multicolumn{2}{|c|}{ Documentos } \\
\hline & & & & & \multirow{2}{*}{$\begin{array}{r}\mathbf{n} \\
223 \\
\end{array}$} & \multirow{2}{*}{$\begin{array}{c}\% \\
3,47 \%\end{array}$} \\
\hline $1^{\mathrm{o}}$ & $\begin{array}{l}\text { Journal of Academic } \\
\text { Librarianship }\end{array}$ & & & & & \\
\hline $2^{\circ}$ & $\begin{array}{l}\text { Communications in } \\
\text { Computer And Information }\end{array}$ & Proceedings Paper (185) & Alemanha & Springer & 185 & $2,88 \%$ \\
\hline $3^{\circ}$ & $\begin{array}{l}\text { Portal-Libraries and The } \\
\text { Academy }\end{array}$ & $\begin{array}{l}\text { Articles (92) } \\
\text { Book Review (18) } \\
\text { Review (1) } \\
\text { Editorial material (1) }\end{array}$ & EUA & $\begin{array}{l}\text { Johns } \\
\text { Hopkins } \\
\text { University } \\
\text { Press }\end{array}$ & 112 & $1,74 \%$ \\
\hline \multirow[t]{3}{*}{$4^{\circ}$} & $\begin{array}{l}\text { College \& Research } \\
\text { Libraries }\end{array}$ & $\begin{array}{l}\text { Articles (89) } \\
\text { Book Review (18) } \\
\text { Editorial material (4) } \\
\text { Review (1) }\end{array}$ & EUA & ACRL & 111 & $1,72 \%$ \\
\hline & Comunicar & $\begin{array}{l}\text { Articles (106) } \\
\text { Editorial material (5) }\end{array}$ & Espanha & $\begin{array}{c}\text { Grupo } \\
\text { Comunicar }\end{array}$ & 111 & $1,72 \%$ \\
\hline & $\begin{array}{l}\text { Journal of Adolescent } \\
\text { Adult Literacy }\end{array}$ & $\begin{array}{l}\text { Articles (81) } \\
\text { Editorial material (15) } \\
\text { Book Review (5) } \\
\text { Review (2) }\end{array}$ & EUA & Wiley & 103 & $1,6 \%$ \\
\hline $6^{\circ}$ & Edulearn Proceedings & Proceedings Paper (98) & Espanha & IATED & 98 & $1,52 \%$ \\
\hline $7^{\circ}$ & Inted Proceedings & Proceedings Paper (89) & Espanha & IATED & 89 & $1,38 \%$ \\
\hline $8^{\circ}$ & $\begin{array}{l}\text { Worldwide Commonalities } \\
\text { And Challenges in } \\
\text { Information Literacy } \\
\text { Research And Practice }\end{array}$ & Proceedings Paper (85) & Alemanha & $\begin{array}{l}\text { Springer- } \\
\text { Verlag }\end{array}$ & 85 & $1,32 \%$ \\
\hline $9^{\circ}$ & $\begin{array}{l}\text { Procedia Social and } \\
\text { Behavioral Sciences }\end{array}$ & Proceedings Paper (82) & $\begin{array}{l}\text { Países } \\
\text { Baixos }\end{array}$ & Elsevier & 82 & $1,27 \%$ \\
\hline $10^{\circ}$ & $\begin{array}{l}\text { Information Research-an } \\
\text { International Electronic } \\
\text { Journal }\end{array}$ & $\begin{array}{l}\text { Articles (71) } \\
\text { Proceedings Paper (40) } \\
\text { Book Review (5) } \\
\text { Review (3) } \\
\text { Editorial material (1) }\end{array}$ & Inglaterra & $\begin{array}{l}\text { Univ. } \\
\text { Sheffield }\end{array}$ & 80 & $1,24 \%$ \\
\hline $11^{\circ}$ & Journal of Documentation & $\begin{array}{l}\text { Articles (63) } \\
\text { Book Review (6) } \\
\text { Review (3) } \\
\text { Editorial material (1) }\end{array}$ & Inglaterra & $\begin{array}{l}\text { Emerald } \\
\text { Group }\end{array}$ & 73 & $1,13 \%$ \\
\hline $12^{\circ}$ & $\begin{array}{l}\text { Information Literacy: } \\
\text { Lifelong Learning and } \\
\text { Digital Citizenship In The } \\
\text { 21st Century }\end{array}$ & Proceedings Paper (72) & Alemanha & $\begin{array}{l}\text { Springer- } \\
\text { Verlag }\end{array}$ & 72 & $1,12 \%$ \\
\hline $13^{\circ}$ & $\begin{array}{l}\text { Reference \& User Services } \\
\text { Quarterly }\end{array}$ & $\begin{array}{l}\text { Articles (45) } \\
\text { Book Review (24) } \\
\text { Editorial material (1) }\end{array}$ & EUA & ALA & 70 & $1,09 \%$ \\
\hline $14^{\circ}$ & $\begin{array}{l}\text { Journal of Librarianship } \\
\text { And Information Science }\end{array}$ & $\begin{array}{l}\text { Articles (57) } \\
\text { Book Review (10) } \\
\text { Review (1) }\end{array}$ & Inglaterra & \begin{tabular}{|c|} 
Sage \\
Publication \\
$\mathrm{s}$
\end{tabular} & 68 & $1,06 \%$ \\
\hline
\end{tabular}


Em rankings anteriores, também se destacaram: Information ResearchAn International Electronic Journal (KUMARI; MADHUSUDHAN; ALI, 2015), Journal of Documentation (KUMARI; MADHUSUDHAN; ALI, 2015; KOLLE, 2017), Reference \& User Services Quarterly (DUDZIAK, 2010), Journal of Librarianship and Information Science (DUDZIAK, 2010; KUMARI; MADHUSUDHAN; ALI, 2015), igualmente listados no ranking da Tabela 3.

Pode-se observar o volume de documentos publicados em eventos e sua relevância para a área pela quantidade de fontes publicadoras exclusivamente deste tipo de documento no ranking da Tabela 3: nas $2^{\mathrm{a}}, 6^{\mathrm{a}}, 7^{\mathrm{a}}, 8^{\mathrm{a}}, 9^{\mathrm{a}}$ e $12^{\mathrm{a}}$ posições. Além disso, este formato também é identificado nos títulos de periódicos, nas $1^{\mathrm{a}}$ e $10^{\mathrm{a}}$ posições, o que reitera sua relevância para as comunicações sobre CoInfo.

Quanto à identificação da origem dos documentos, a partir da análise da autoria e dos vínculos institucionais e nacionais dos autores foram identificadas 10.531 notações diversificadas de autoria, que incluem 18 registros anônimos. Estes autores somam 13.745 autorias em publicações, uma média de 2,14 autores por documento, superior à média de índice de colaboração observada por Pinto, Escalona-Fernández e Pulgarín (2013), de 1,87 autores por artigo.

Em geral, a produtividade dos autores é baixa, a maioria, 8.843 (83,97\%), publicou apenas um trabalho, $12,12 \%$ (1.666 autores) publicou entre 2 a 10 trabalhos (1.666) e, os $0,15 \%$ (21) mais produtivos assinam de 11 a 40 documentos. Estes últimos assinam a autoria de 349 (5,42\%) documentos, uma média de 16,62 documentos por autor, indicando um núcleo de autores na temática.

O autor com maior produtividade no tema é Maria Pinto, que publicou 40 documentos e possui vínculo com a Universidade de Granada, na Espanha. A autora possui 13 trabalhos de diferença do segundo colocado no ranking (Quadro 1), sendo também identificada como a mais produtiva na temática nos estudos de Bhardwaj (2017), Kolle (2017), Majid et al. (2015) e Pinto, Escalona-Fernández e Pulgarín (2013). A segunda colocada, Heide Julien, filiada à State University of New York at Buffalo, nos EUA, é autora de 27 dos 
documentos sobre CoInfo, e é identificada nos estudos de Dudziak (2010) e Kumari Ali e Madhusudhan (2015) como a autora mais citada na temática, seguida por Maria Pinto. AnneMaree Lloyd, Christine Bruce e Ina Fourie também aparecem nas $3^{\mathrm{a}}, 4^{\mathrm{a}}$ e $5^{\mathrm{a}}$ colocações do estudo de Kumari, Ali e Madhusudhan (2015).

Quadro 1 - Ranking dos autores mais produtivos sobre CoInfo

\begin{tabular}{|c|c|c|c|c|}
\hline \multicolumn{2}{|c|}{$\mathrm{N}^{0}$ documentos } & Autor & Instituição & País \\
\hline $1^{\mathrm{o}}$ & 40 & Pinto, M. & University of Granada & Espanha \\
\hline $2^{\circ}$ & 27 & Julien, $\mathrm{H}$ & $\begin{array}{l}\text { State University of New York at } \\
\text { Buffalo }\end{array}$ & EUA \\
\hline $3^{\circ}$ & 21 & Lloyd, A & University of Boras & Suécia \\
\hline \multirow{2}{*}{$4^{\circ}$} & \multirow{2}{*}{18} & Bruce, $\mathrm{C}$ & $\begin{array}{lll}\text { Queensland } & \text { University } & \text { of } \\
\text { Technology } & & \end{array}$ & Austrália \\
\hline & & Majid, S. & Nanyang Technology University & Cingapura \\
\hline \multirow{3}{*}{$5^{\circ}$} & \multirow{3}{*}{16} & Foo, $\mathrm{S}$ & Nanyang Technology University & Cingapura \\
\hline & & Fourie, I & University of Pretoria & África do Sul \\
\hline & & Primack, BA & University of Pittsburgh & EUA \\
\hline \multirow{2}{*}{$6^{\circ}$} & \multirow{2}{*}{15} & Fosmire, $\mathrm{M}$ & Purdue University & EUA \\
\hline & & Hobbs, R & University Rhode Island & EUA \\
\hline \multirow{2}{*}{$7^{\circ}$} & \multirow{2}{*}{14} & Austin, EW & Washington State University & EUA \\
\hline & & Koltay, T & Eszterhazy Karoly University & Hungria \\
\hline \multirow{2}{*}{$8^{\circ}$} & \multirow{2}{*}{12} & Mokhtar,IA & National Institute of Education & Cingapura \\
\hline & & Wade, TD & Flinders University & Austrália \\
\hline \multirow{7}{*}{$9^{\circ}$} & \multirow{7}{*}{11} & Bawden, D & University of London & Inglaterra \\
\hline & & Brage, $\mathrm{C}$ & $\begin{array}{lcc}\text { International } & \text { Association } & \text { of } \\
\text { Technology } & \text { Education } & \& \\
\text { Development } & \text { (IATED) } & \\
\end{array}$ & Espanha \\
\hline & & Fedorov, A & Rostov State University & Rússia \\
\hline & & Hargittai, E & University Zurich & Suíça \\
\hline & & Paxton, SJ & La Trobe University & Austrália \\
\hline & & Saunders, L & Simmons College & EUA \\
\hline & & Wilksch, SM & Flinders University & Austrália \\
\hline
\end{tabular}

Fonte: dados da pesquisa.

Pode-se observar que há ampla diversidade de instituições e nacionalidades no ranking de autoria. Existem duas instituições que se repetem no ranking de autores, a Flinders University, sediada na Austrália, com autores na sétima e oitava posição, em um total de 23 documentos, e a Nanyang 
Technology University, de Singapura, que possui autores nas terceiras e quartas posições, totalizando 34 documentos publicados.

Dentre os 21 autores mais produtivos, 6 possuem vínculo com instituições norte-americanas, 4 australianas, 3 de Singapura e 2 da Espanha, de acordo com o Quadro 1. Neste ranking, aparecem ainda autores de instituições sediadas na Inglaterra, Hungria, Rússia, África do Sul, Suécia e Suíça.

Entre as instituições mencionadas nos vínculos dos autores, foram identificadas 3.360 notações diversas, utilizado o Campo Organizações Aprimorada (Organization - Enhanced) em WoS, que retoma todos os registros que contêm o nome preferencial da instituição e todos os registros que contêm variantes do nome, como abreviaturas, por exemplo (CLARIVATE ANALYTICS, 2018) $)^{2}$. A partir da soma dos documentos vinculados individualmente a cada uma destas instituições, 10.477, se obtém uma média de 3,12 documentos vinculados a cada instituição, embora 62,56\% (2.102) delas estejam vinculadas a apenas um documento.

As 16 instituições $(0,47 \%)$ com maior números de menções, superior a 50 documentos, compõem o ranking das instituições com o maior número de documentos publicados. Destas, 12 são norte-americanas, incluindo as cinco primeiras colocadas no ranking, dentre as quais Pennsylvania Commonwealth System Of Higher Education, ocupa a primeira posição com um total de 95 documentos publicados (Tabela 5).

Tabela 5 - Ranking das instituições mais produtivas sobre CoInfo

\begin{tabular}{|c|c|c|c|}
\hline \multirow{2}{*}{\multicolumn{2}{|c|}{ Instituição }} & \multicolumn{2}{|c|}{ Documentos } \\
\hline & & \multirow{2}{*}{$\begin{array}{c}\mathbf{n} \\
95\end{array}$} & \multirow{2}{*}{$\begin{array}{c}\% \\
1,48 \%\end{array}$} \\
\hline $1^{\circ}$ & Pennsylvania Commonwealth System Of Higher Education & & \\
\hline $2^{\circ}$ & California State University System & 88 & $1,37 \%$ \\
\hline $3^{\circ}$ & University Of California System & 83 & $1,29 \%$ \\
\hline $4^{\circ}$ & University Of North Carolina & 79 & $1,23 \%$ \\
\hline $5^{\circ}$ & State University System Of Florida & 67 & $1,04 \%$ \\
\hline $6^{\circ}$ & Nanyang Technological University & 64 & $0,99 \%$ \\
\hline $7^{\circ}$ & University of Texas System & 59 & $0,92 \%$ \\
\hline \multirow{3}{*}{$8^{\circ}$} & Indiana University System & 57 & $0,89 \%$ \\
\hline & University Of Granada & 57 & $0,89 \%$ \\
\hline & University Of London & 57 & $0,89 \%$ \\
\hline $9^{\circ}$ & Purdue University System & 56 & $0,87 \%$ \\
\hline $10^{\circ}$ & City University Of New York Cuny System & 54 & $0,84 \%$ \\
\hline
\end{tabular}




\begin{tabular}{l|l|l|l} 
& Queensland University Of Technology & 54 & $0,84 \%$ \\
& University Of Illinois System & 54 & $0,84 \%$ \\
$11^{\circ}$ & University System Of Georgia & 54 & $0,84 \%$ \\
\hline
\end{tabular}

Fonte: Dados da pesquisa.

Constam, no ranking, uma instituição australiana, a Queensland University of Technology, uma inglesa, University of London, uma espanhola, University of Granada e uma singapurense, a Nanyang Technological University, ambas associadas aos autores mais produtivos na temática (Quadro $1)$.

Estes resultados demonstram a forte presença das instituições norteamericanas (Tabela 5) na publicação de documentos sobre CoInfo, assim como a tendência da base na cobertura de estudos de origem estadunidense (Tabela 1), evidenciada na autoria (Quadro 1) e na participação de instituições estadunidenses na produção e, também na edição das fontes de informação (Tabela 3) indexadas na base. Entretanto, os resultados ainda apontam, embora tímida, a presença crescente de nacionalidades variadas na produção científica sobre CoInfo indexada em WoS (Tabela 1), sinalizando a expansão geográfica da produção indexada na base sobre a temática.

\section{Considerações finais}

O campo de pesquisa em CoInfo tem crescido e se expandido nas últimas duas décadas, tendo quase triplicado de volume nos últimos cinco anos (Figura 2) e acumulando um maior impacto a cada ano (Figura 3), conforme é possível observar nos resultados deste estudo. Ao resgatar a evolução histórica da CoInfo como fenômeno social e como campo de estudos, pela análise de sua produção científica ao longo do tempo, este estudo permite não somente a identificação dos padrões de publicação e a origem da produção científica publicada entre os periódicos de maior prestigio mundial, mas proporciona a identificação de lacunas na representação científica de origem diversificada sobre a temática e de nichos de atuação. 
De modo geral, as características da produção científica indexada em WoS têm se mantido dentro dos padrões das bases de dados internacionais: artigos em língua inglesa originários de países desenvolvidos, com participação majoritária dos EUA e publicados em fontes de informação editadas neste país (Tabelas 1 e 3). Estes resultados reforçam o elitismo da base utilizada, considerada a fonte de informação científica com melhor prestígio, assim como evidenciam a consolidação da CoInfo como um campo de estudo à medida que cresce o volume de publicações na temática e sua representação no universo limitado e prestigiado da WoS.

Apesar disso, é possível observar um movimento - ainda que sutil - de popularização da disciplina em outros países e de representação da produção científica destes na WoS pela sua menção e inclusão em alguns dos rankings de produtividade sobre a temática (Tabela 1 e Quadro 1). Assim, esses resultados também apontam a necessidade de estudos mais aprofundados, que empreguem o uso de fontes de informações diversificadas concomitantemente, para a obtenção de um panorama global acerca do desenvolvimento mundial das pesquisas sobre CoInfo, que incluam, por exemplo, publicações dos estudos desenvolvidas em regiões consideradas periféricas, como a América Latina.

\section{Referências}

AHARONY, N. Information literacy in the professional literature: an exploratory analysis. Aslib Proceedings: New Information Perspectives, Bristol, v. 62, n. 3, p. 261-282, 2010. Disponível em: https://www.emeraldinsight.com/doi/full/10.1108/00012531011046907. Acesso em 03 maio 2020.

AMERICAN LIBRARY ASSOCIATION. Presidential Committee on Information Literacy: final report. Washington, 1989. Disponível em: http://www.ala.org/acrl/publications/whitepapers/presidential. Acesso em: 03 maio 2020.

ASSOCIATION OF COLLEGE AND RESEARCH LIBRARIES. Framework for Information Literacy for Higher Education. Chicago: American Library Association, 2016. Disponível em:

http://www.ala.org/acrl/sites/ala.org.acrl/files/content/issues/infolit/Framework_ ILHE.pdf. Acesso em: 03 maio 2020. 
BHARDWAJ, R. K. Information Literacy Literature in the Social Sciences and Humanities: a bibliometric study. Information and Learning Science, London, v. 118, n. 1-2, p. 67-89, 2017. Disponível em:

https://www.emeraldinsight.com/doi/abs/10.1108/ILS-09-2016-0068. Acesso em: 03 maio 2020.

BREIVIK, P. S. Putting libraries back in the information society. American Libraries, Chicago, v. 16, n. 1, 1985. Disponível em: https://www.questia.com/read/1G1-4003694/putting-libraries-back-in-theinformation-society. Acesso em: 03 maio 2020.

BURCHINAL, L. G. The Communication Revolution: America's Third Century Challenge. College Station (Texas): A\&M University Library, 1976. Disponível em: https://personalpages.manchester.ac.uk/staff/drew.whitworth/burchinal_the_com munications_revolution.pdf. Acesso em: 03 maio 2020.

CLARIVATE ANALYTICS. Web of Science. [s.1.], 2018. Disponível em: <www.webknowledge.com>. Acesso em 03 maio 2020.

DUDZIAK, E.A. A information literacy e o papel educacional das bibliotecas. 2001. Dissertação (Mestrado em Ciências da Comunicação) Escola de Comunicação e Artes, Universidade do estado de São Paulo, São Paulo, 2001. Disponível em: http://www.teses.usp.br/teses/disponiveis/27/27143/tde-30112004-151029/ptbr.php. Acesso em: 03 maio 2020.

DUDZIAK, E.A. Information Literacy: princípios, filosofia e prática. Ciência da Informação, Brasília, v. 32, n. 1, p. 23-35, jan./abr. 2003. Disponível em: http://revista.ibict.br/ciinf/article/view/1016/1071. Acesso em: 03 maio 2020.

DUDZIAK, E. A. Competência Informacional: análise evolucionária das tendências da pesquisa e produtividade científica em âmbito mundial.

Informação \& Informação, Londrina, v. 15, n. 2, p. 1-22, jul./dez. 2010. Disponível em:

http://www.uel.br/revistas/uel/index.php/informacao/article/view/7045. Acesso em: 03 maio 2020.

GONZÁLEZ DE GÓMEZ, M. N. Novos cenários políticos para a informação. Ciência da Informação, Brasília, v. 31, n. 01, p. 27-40, jan./abr. 2002. Disponível em: http://revista.ibict.br/ciinf/article/view/975. Acesso em: 03 maio 2020.

GONZÁLEZ-VALIENTE, C.L. La investigación cubana sobre alfabetización informacional: un análisis de su literatura publicada. Revista Cubana de Información en Ciencias de la Salud, Habana, v. 25, n. 4, p. 426-441, 2014. Disponível em: http://scielo.sld.cu/scielo.php?script=sci_arttext\&pid=S230721132014000400006. Acesso em: 03 maio 2020. 
HAMELINK, C. An alternative to news. Journal of Communication, Oxônia, v. 26, n. 4, p. 120-123, 1976. Disponível em:

https://onlinelibrary.wiley.com/doi/abs/10.1111/j.1460-2466.1976.tb01947.x_. Acesso em: 03 maio 2020.

INTERNATIONAL FEDERATION OF LIBRARIES ASSOCIATIONS AND INSTITUTIONS. Declaração de Alexandria sobre competência

Informacional e aprendizado ao longo da vida. In: National Fórum on Information Literacy, Alexandria, 2005. Disponível em:

$<$ https://www.ifla.org/publications/beacons-of-the-information-society-thealexandria-proclamation-on-information-literacy>. Acesso em: 03 maio 2020.

KOLLE, S. R. Global Research on Information Literacy: a bibliometric analysis from 2005 to 2014. The Eletronic Library, London, v. 35, n. 2, p. 283-298, 2017. Disponível em: https://www.emeraldinsight.com/doi/abs/10.1108/EL-082015-0160. Acesso em: 03 maio 2020.

KUHLTHAU, C. C. Information Skills for an Information Society: a review of research. Nova York: ERIC Clearing house on Information Resources, 1987. Disponível em: https://files.eric.ed.gov/fulltext/ED297740.pdf. Acesso em: 03 maio 2020.

KUMAR, K. A Scientometric study of digital literacy in online library information science and technology abstracts (LISTA). Library Philos and Practice, Lincoln, jan. 2014. Disponível em:

http://digitalcommons.unl.edu/libphilprac/1044. Acesso em 03 maio 2020.

KUMARI, A.; MADHUSUDHAN, C.M; ALI, H. A bibliometric study of world research output on information literacy in the field of library and information science during 1999-2013. E-Library Science Research Journal, Maharashtra, v._3, n._9, p. 1-10, 2015. Disponível em:

http://oldlsrj.lbp.world/ArticleDetails.aspx?id=507. Acesso em 03 maio 2020.

LEITE, C. et al. Cenário e perspectiva da produção científica sobre competência em informação (CoInfo) no Brasil: estudo da produção no âmbito da ANCIB.

Informação e Sociedade: estudos, João Pessoa, v. 26, n. 3, p. 151-168, set./dez. 2016. Disponível em:

https://periodicos.ufpb.br/ojs2/index.php/ies/article/view/31983/16876 . Acesso em: 03 maio 2020.

MACIAS-CHAPULA, C. A. O papel da infometria e da cienciometria e sua perspectiva nacional e internacional. Ciência da Informação, Brasília, v. 27, n. 2, p. 134-140, maio/ago. 1998. Disponível em:

http://www.scielo.br/pdf/ci/v27n2/2729805.pdf. Acesso em: 03 maio 2020.

MAJID, S. et al. Analyzing publishing trends in information literacy literature: a bibliometric study. Malaysian Journal of Library and Information Science, Kuala Lumpur, v. 20, n. 2, p. 51-66, 2015. Disponível em: 
https://ejournal.um.edu.my/index.php/MJLIS/article/download/1766/2530 . Acesso em: 03 maio. 2020.

PINTO, M.; CORDÓN, J. A.; DÍAZ, R. G. Thirty years of information literacy (1977-2007): a terminological, conceptual and statistical analysis. Journal of Librarianship and Information Science, [s.1], v. 42, n. 1, p. 3-19, Mar. 2010. Disponível em:

http://journals.sagepub.com/doi/pdf/10.1177/0961000609345091. Acesso em: 03 maio 2020.

PINTO, M.; ESCALONA-FERNÁNDEZ, M. I.; PULGARÍN, A. Information Literacy in social sciences and health sciences: a bibliometric study.

Scientometrics, Budapest, v. 95, n. 3, p. 1071-1094, Jun 2013. Disponível em: https://link.springer.com/article/10.1007/s11192-012-0899-y . Acesso em: 03 maio 2020.

RADER, H. Information Literacy 1973-2002: a selected literature review.

Library Trends, Baltimore, v. 51, n. 2, p. 242-259, May/Aug. 2002. Disponível em:https://pdfs.semanticscholar.org/4bd4/3dbfef43ac315ade1ae8bd43147a174e c754.pdf. Acesso em: 03 maio 2020.

SARACEVIC, T. Ciência da Informação: origem, evolução e relações.

Perspectivas em Ciência da Informação, Belo Horizonte, v. 1, n. 1, 1996.

Disponível em:

http://portaldeperiodicos.eci.ufmg.br/index.php/pci/article/view/235. Acesso em: 03 maio 2020.

VITORINO, E. V.; PIANTOLA; D. Competência Informacional - bases históricas e conceituais: construindo significados. Ciência da Informação, Brasília, v. 38, n. 3, p. 130-141, set/dez. 2009. Disponível em: http://www.scielo.br/pdf/ci/v38n3/v38n3a09.pdf. Acesso em: 03 maio 2020.

ZURKOWSKI, P. Information services environment: relationships and priorities. Washington: National Commission on Libraries, 1974. Disponível em: https://files.eric.ed.gov/fulltext/ED100391.pdf. Acesso em: 03 maio 2020.

\title{
World scientific production in information literacy: analysis of indexed documents in Web of Science
}

\begin{abstract}
This is a research that comprises the measurement of scientific production on information literacy worldwide, that is suitable for obtaining indicators about the scientific activity of the discipline. Analyzes the world scientific production of information literacy indexed in Web of Science, from 1957 to 2017. The study's objectives are: a) identify the standards of scientific production of information literacy, in what it refers to type of publishing and their access model, year, citation, language, area and publication vehicles and,
\end{abstract}


b) identify the origin of the published documents, based on authorship, institutional link and the nationality of the author. The research universe consists of the documents indexed in Web of Science referred to Information Literacy, in all formats and languages published until 2017, totaling 6.634 documents. The results demonstrate the exponential growth of the number of documents of the theme in the last decade, concentrating $74.85 \%$ of the total documents, with the predominance of publications in the format of articles $(62.69 \%)$, published in English (90.43\%) in the areas Information Science (34.96\%) and education (34\%), by authors from 112 countries. In the end, it signals the numerical and geographical expansion in the amount of documents published and indexed on Information Literacy in the Web of Science.

Keywords: Information Literacy. Scientific Production. Web of Science

Recebido: 19/09/2019

Aceito: 26/12/2019

\section{Declaração de autoria}

Concepção e elaboração do estudo: Patrícia da Silva Neubert, Djuli Machado de Lucca

Coleta de dados: Patrícia da Silva Neubert

Análise e discussão de dados: Patrícia da Silva Neubert, Djuli Machado de Lucca

Redação e revisão do manuscrito: Patrícia da Silva Neubert, Djuli Machado de Lucca

\section{Como citar}

DE LUCCA, Djuli Machado; NEUBERT, Patricia da Silva. A produção científica mundial sobre Competência em Informação: análise dos documentos indexados na Web of Science. Em Questão, Porto Alegre, v. _, n. _, p. _-_, set./dez. 2020. DOI: https://doi.org/10.

${ }^{1}$ Entre 1957 e 1974 - ano em que o termo information literacy é cunhado - os documentos sobre a temática apresentam os termos media literacy e information learning.

${ }^{2}$ É importante observar que este campo não é absolutamente consistente, podendo haver variações de nomes das instituições grafados de outras formas não contemplados no agrupamento. 